\title{
Jürgen Fohrmann
}

\section{DAS ANDERE DER, KulTuR': Die, KULTUR' DER KULTURWISSENSCHAFTEN}

I.

Die Vorstellung sozialer Räume und kultureller Praktiken hängt aufs engste zusammen mit der dispersen oder einheitsstiftenden Kraft, die dem Kulturbegriff selbst zugemutet wird. Die nachfolgenden Überlegungen konzentrieren sich daher darauf, zunächst am Beispiel dreier Zäsuren die konzeptuellen Umformulierungen dieses Kulturbegriffs im Rahmen der vornehmlich europäischen Diskussion nachzuzeichnen, um dann am Ende noch einmal die Bezogenheit von ,sozialem Raum' und ,kultureller Praxis' auch in ihrer politischen Dimension in den Blick zu nehmen.

\section{II.}

„Ohne Cultur war und ist der Mensch nicht etwa nur ein rohes Holz, ein ungeformter Marmor, sondern er ist und wird ein brutum. “"

Wir verdanken es vornehmlich Reinhart Koselleck, auf den Kollektivsingular im letzten Drittel des 18. Jahrhunderts aufmerksam geworden zu sein. ${ }^{2}$

Dieser Singular setzt sich auch durch im Falle der ,Cultur', die nun ohne Genitivattribut erscheinen kann, also nicht mehr nur die Kultur des Bodens oder die cultura animi ist, sondern jetzt für sich allein zu stehen vermag: ,Cultur' wird eine Summenformel, und in ihr vereinheitlichen sich die ,Culturen' zu der, Cultur'.

,Cultur' rückt damit ein in die Reihe absolut gebrauchter Termini und wird in diesem Sinne universalisiert wie die Kollektivbegriffe ,Geschichte' oder ,Natur' - absolut sind sie, weil ihre Extension alles zu umfassen sucht und weil sie damit alle Gegenbegriffe integrieren. Denn was wäre nicht ,Natur', was nicht ,Geschichte' - und was wäre nicht ,Cultur'? Ist es nicht auch just der Vorwurf, der dann - aus dem 18. Jahrhundert transferiert - die heutige Kulturwissenschaft einholt, scheint sie doch alles sein zu wollen und damit

1 Johann Gottfried Herder (SWS 22, 310). Vgl. Marion Heinz: „Kulturtheorien der Aufklärung: Herder und Kant", in: Regine Otto (Hg.), Nationen und Kulturen. Zum 250. Geburtstag Johann Gottfried Herders, Würzburg 1996, S. 139-152.

2 Vgl. Reinhart Koselleck: Vergangene Zukunft. Zur Semantik geschichtlicher Zeiten, 4. Aufl., Frankfurt/Main 1985. 
dann nichts zu werden? Denn die Einheit aller Differenzen ist leer wie voll zugleich, ist nichts und alles, und beides kann nicht mehr unterschieden werden.

Nun ist aber schon aus der oben angeführten Herder-Stelle ersichtlich, daß solche absoluten Begriffe - vielleicht außer: Gott, aber der bleibt ja auch eine Ausnahme - zwar programmatisch existieren mögen, gleichwohl sich im Gefüge der Aussagen aber wieder über Unterschiede, die sie implizieren, überhaupt erst ergeben. Sie sind die eine Seite einer Unterscheidung, deren andere Seite meist - um einen absoluten Begriff zu erhalten - ,verdunkelt ${ }^{\star}$ wird (wenn diese aufklärerische Hinsicht gestattet ist). Bei Herder ist die andere Seite der ,Cultur' das schlechthin Rohe, das brutum, das Ungeformte, das zugleich Rohstoff wie auch archaischen Schrecken bedeutet. Rohstoff ist es, weil ein Pygmalion kommen mag, der dem Marmor Form gibt, ihn ,kulturiert ${ }^{\star}$ und damit überhaupt erst - in mehrfacher Bedeutung des Wortes - ,bildet'. Die alte Unterscheidung ,barbari - eruditi' wird hier, wie genauer zu sehen wäre, in eine Metaphysik des Lebenspendens transformiert, die den Initialpunkt für einen perfekten oder auch unendlich perfektiblen Bildungsprozess abgibt. Nimmt man also - und sei es auch zunächst nur hypothetisch - an, dass es absolute Begriffe nicht gibt, dann ist es gar nicht beliebig, sondern höchst bedeutsam, sich der in der Regel verdeckten Seite der Unterscheidung zuzuwenden. Dies soll für die ,Kultur', die in der Rede von heutiger ,Kulturwissenschaft' impliziert wird, in einer groben Skizze zu entfalten versucht werden. Gewählt wird dabei der Weg historischer Analyse: Sehr knapp sollen drei Phasen der Rede über ,Kultur' rekonstruiert werden, nicht, um eine kohärente Verlaufsgeschichte zu bieten, sondern um Prozesse der Anreicherung und Umpolung darstellen zu können. Dass es sich hierbei weitgehend um die Säkularschnitte ,um 1800‘, um 1900‘ und ,um 2000` handelt, hat weniger mit Symmetrie oder Bedeutsamkeit, sondern eher mit einer bewusst herbeigeführten Übersichtlichkeit zu tun.

\section{III.}

Eine kurze Rekapitulation sei an den Anfang gestellt. ${ }^{3}$ Bei allen nötigen Differenzierungen und Überschneidungen lassen sich doch zwei Traditionen, die die Konzepte von ,Cultur' dann seit dem 18. Jahrhundert bestimmen, auseinander halten: Dies ist einmal der Rekurs auf die Polis-Konzeption der griechischen Antike und hier besonders die tentative Entgegensetzung von Freiheit und körperlicher Arbeit. Und das ist andererseits die römische Tradition vieler ,culturae', die Pflege, Bebauung usw. in körperlicher und geistiger Hinsicht verstand und sie sowohl mit ,industria' koppelte als auch in ihr keinen Gegensatz zum ,civis` zu sehen schien.

3 Vgl. dazu Georg Bollenbeck: Bildung und Kultur. Glanz und Elend eines deutschen Deutungsmusters, Frankfurt/Main 1994. 
Springt man ins (deutsche) 18. Jahrhundert, so ist zu beobachten, dass diese ,römische' Konzeption vieler ,culturae' den ,Cultur'-Begriff der Kameralisten, der Popularphilosophen, der Philantropen und insbesondere der Universalhistoriker mit ihrer, Culturgeschichte“ prägte. Hier wird das Ziel verfolgt, eine umfassende Poliziertheit der Gattung auf der Grundlage entfalteten Fleisses zum Ziel der Menschheit zu erklären. Dieser Begriff der ,Cultur' ist sehr eng angelehnt an die französische Auffassung von ,civilisation“.

Die ,griechische Konzeption“ hingegen, und dies ist eine vornehmlich für das deutsche 19. Jahrhundert einschneidende Entwicklung, wird dann insbesondere vom Neuhumanismus aufgegriffen, als ,Bildungsvorstellung' ausformuliert und Schritt für Schritt, ganz konsequent, gegen einen nun als defizient erscheinenden Begriff von ,Zivilisation“ abgegrenzt.

Kultur als Effekt von ,Bildung' mag zwar sub specie aeterni auch auf ein Gattungswesen teleologisch ausgerichtet sein, wird aber im wesentlichen vom einzelnen Subjekt, von der Person, aus gedacht. Kultur als Bildung zielt damit - aller Formalisierungen durch Erziehung zum Trotz - auf ein individuelles, nicht klar steuerbares Programm, dem kein Präfix lieber ist als ,selbst-‘.

In gewisser Weise holt der Neuhumanismus - zumindest in der Humboldtschen Variante - damit die Uminterpretation des Historischen, die von Vico am entschiedensten vorgenommen wurde, auf der Ebene des Subjekts ein: Das Gewisse, das certum als das hypostasierte Wahre, verum, wird zum zentralen Gegenstand menschlicher Beobachtung, soweit sie die Geschichte, mithin die menschlichen Angelegenheiten, betrifft, und solche Beobachtung leistet für Vico die Philologie: ,[...] die Philologie beobachtet, was die menschliche Willkür als Gesetz aufgestellt hat, und daraus entsteht das Bewußtsein von dem, was gewiß ist. “4

,Humanitas' leitet Vico in diesem Sinne konsequent aus dem Totenbegräbnis ab und denkt dabei sowohl an die Erinnerung als auch an die Teilung der Felder, an Zu-Teilung. In diesem Sinne ist Kultur als ,Bildung ' zugeteilte Kenntnis der Tradition, eine memoria, die Geschichte und Gegenwart so verbindet, dass vergangene Welt zunächst im Subjekt eingeschlossen und dann von innen heraus (immer wieder) neu gebildet wird. Kultur entsteht daher, weil ein inkorporierendes Inneres so spricht, dass ein anderes Inneres angesprochen, apostrophiert werden kann und erwidert, und zwar idealerweise in komplementärer Funktion. Gedacht ist an die unendliche Zwiesprache geselliger Wesen, die ein Soziales ergibt. Dieses Soziale hat sowohl im Neuhumanismus die Rousseausche Vorstellung von der Indifferenz im Naturzustand als auch die Rousseausche Dialektik von Eigentumsumgrenzung und Aufeinander-Angewiesen-Sein hinter sich gelassen. Sozialität ist die (fingiert) mündliche Kultur affiner Personen. Alles andere ist Zivilisation, alte "Höfischkeit', etwas Oberflächliches, Zweites, nicht die Stimme von innen, sondern nur ihr Echo, ist, wie es dann bald in und nach den Befreiungskriegen heißen wird, Frankreich, das Äußerliche, von dem kein Weg nach innen zurückführt.

4 Giambattisto Vico: Die neue Wissenschaft über die gemeinschaftliche Natur der Völker. Nach der Ausgabe von 1744, übersetzt und eingeleitet von Erich Auerbach, Berlin 1965, S. 78. 
IV.

Um 1900 - oder doch etwas genauer: 1883 - formuliert Wilhelm Dilthey dann: „Die Natur ist uns stumm. Nur die Macht unserer Imagination ergießt einen Schimmer von Leben und Innerlichkeit über sie. [...] Die Natur ist uns fremd. Denn sie ist nur ein Außen, kein Inneres. ${ }^{65}$,Frankreich“ wird so zur mechanischen Natur, das Äußerliche zum Außen.

Spätestens zwischen 1880 und den 1920er Jahren vollzieht sich eine gravierende Transformation des neuhumanistischen Kulturkonzepts. In ihr wird die Frontstellung gegen ein nur ,Äußerliches“, die sowohl aus der Kritik an einem französischen Zivilisationsanspruch als auch aus der Ablehnung einer popularphilosophischen Aufklärung sich speiste, durch einen ontologischen Gegensatz von Innen und Außen überschrieben. Außen heißt jetzt: die naturhafte Ordnung des Wiederholbaren, daher Mechanischen, der gesetzmäßigen Vollzüge in der Natur und, wie manche - für Dilthey zu unrecht - auch meinten, in der Gesellschaft. Dieses Außen wird von den Naturwissenschaften verwaltet, die sich, betrieben durch ihre geisteswissenschaftlichen Agenten, nun anschickten, auch die menschliche Sozialität mit ihrem mechanistischen Modell zu behandeln. Gegen Taine und Buckle betont daher Dilthey:

„Der Strom des Geschehens in [...] [der Gesellschaft] fließt unaufhaltsam voran, während die einzelnen Individua, aus denen er besteht, auf dem Schauplatz des Lebens erscheinen und von ihm wieder abtreten. So findet das Individuum sich in ihm vor, als ein Element, mit anderen Elementen in Wechselwirkung. Es hat das Ganze nicht gebaut, in das es hineingeboren ist. Es kennt von den Gesetzen, in denen hier Individuen aufeinander wirken, nur wenige und unbestimmt gefaßte. “6

Dies ist die geistesgeschichtliche conditio humana: Teil zu sein von etwas, dessen Gesetze man zwar nur unvollkommen kennt, dessen man aber qua Teilhabe ,teilhaftig' werden kann: ,[...] daß ich selber, der ich mich von innen erlebe und kenne, ein Bestandteil dieses gesellschaftlichen Körpers bin, und daß die andern Bestandteile mir gleichartig und sonach für mich ebenfalls in ihrem Innern auffaßbar sind. Ich verstehe das Leben der Gesellschaft. “7

Dies führt dann nicht zu einer Soziologie oder gar Ethnologie, sondern zu einem radikalen Denken der Gesellschaft vom Subjekt aus, ,und grenzenlos ist die Singularität ${ }^{\star 8}$, so grenzenlos, dass es gelingen kann, ,,allem innerhalb der Burgfreiheit [...] [der] Person zu widerstehen."

Dieses Denken des Singulären bei Dilthey, der ja von Geistes- und nicht von Kulturwissenschaften spricht, bildet im Rahmen einer ,Kritik der historischen Vernunft' gleichwohl die Basis für die daraus ableitbaren Kulturbegrif-

5 Wilhelm Dilthey: Einleitung in die Geisteswissenschaften. Versuch einer Grundlegung für das Studium der Gesellschaft und der Geschichte, Bd. 1, Leipzig, Berlin 1922, S. 36.

6 W. Dilthey, Einleitung, S. 36.

7 Ebd., S. 37.

8 Ebd.

9 Ebd, S. 6. 
fe des Neukantianers Heinrich Rickert, auch Ernst Cassirers oder Georg Simmels. Denn bei allen offensichtlichen Unterschieden zwischen diesen Autoren ist Kultur doch im wesentlichen die Semiose eines so verstandenen und auf diese Weise als verstehend begriffenen Subjekts. Der Natur als „Inbegriff des von selbst Entstandenen“ steht auch bei Rickert daher „die Kultur als das von einem nach gewerteten Zwecken handelnden Menschen entweder direkt Hervorgebrachte oder [...] so doch wenigstens um der daran haftenden Werte willen absichtlich Gepflegte gegenüber." ${ }^{10}$ Und noch emphatischer heißt es dann in Simmels „Tragödie der Kultur“: „Kultur ist der Weg von der geschlossenen Einheit durch die entfaltete Vielheit zur entfalteten Einheit. “11

Auch ohne diese eher enigmatische Formulierung an dieser Stelle ganz aufzulösen, wird ersichtlich, dass immer wieder das Singuläre und seine Semiose als Zentrum des Kulturbegriffs zugrundegelegt werden. Dies ist insbesondere bei Cassirer zu sehen, dessen gesamte Rekonstruktion abendländischer Philosophiegeschichte die eine Achse hervorhebt, die bei Herder ihren Ausgang nimmt, dann Wilhelm von Humboldt als Kronzeugen anführt und die in der These gipfelt, dass der Durchbruch zur Kultur mit dem immer auch reflexiven Imperativ des ,Bedeuten-Wollens' ${ }^{`}$ zusammenhänge. Dieses ,Bedeuten-Wollen' sei es, das uns Menschen vom Rest der Natur unterscheide und dessen Entfaltung ein „Leben in Bedeutungen“"12 ergebe. Wenn es den Naturwissenschaften angelegen ist, die „Baupläne ${ }^{\text {"13 }}$ der Natur zu studieren, so sei die Form der Gegenstand einer Wissenschaft von der Kultur. Die Kultur als Form, so lässt sich sagen, ist dieser Vorstellung der Sprachkörper des Subjekts. Damit wird die Singularität der Person, seine Burgfreiheit, durch seine Semiose studierbar, und zwar auch für diese Person selbst. Die Kultur ist mithin die Form, in der sich ein Inneres unverwechselbaren Ausdruck gibt; und sie ist zugleich jenem Anderen entgegengesetzt, das die verwechselbaren, weil sich wiederholenden Naturverläufe zum Gegenstand der (experimentellen) Beobachtung macht. Dies ist die erste Bestimmung der ,anderen“ Seite.

Die zweite Bestimmung (der anderen Seite) ist aber eine Vorstellung von Gesellschaft, die nicht als Körper, genauer: nicht als die Ausdehnung des eigenen Körpers zu betrachten ist. Führte diese rein körperliche Ausdehnung zu einem Modell von Gesellschaft, das wohl eher als Gemeinschaft zu bezeichnen wäre, so richtet sich die Kritik des skizzierten Kulturverständnisses gegen eine Gesellschaft, die von den vermeintlichen Übeln der Moderne, von Industrialisierung, Mechanisierung und von Massenkommunikation angetrieben wird und die - in Anlehnung an Nietzsches Worte - der Teufel und die Statistik holen sollen. Sie entwendet die einzelnen Formen ihren Urhebern, deklariert sie als Waren und will das nicht aufkommen lassen, was Simmel in seiner

10 Heinrich Rickert: Kulturwissenschaften und Naturwissenschaften. Nach der 6. Aufl. von 1926, hg. v. Friedrich Vollhardt, Stuttgart 1986, S. 35.

11 Georg Simmel: „Der Begriff und die Tragödie der Kultur“, in: Ralf Konersmann (Hg.), Kulturphilosophie, Leipzig 1996, S. 25-57, hier S. 27.

12 Ernst Cassirer: „Der Gegenstand der Kulturwissenschaft“, in: ders., Zur Logik der Kulturwissenschaften, Darmstadt 1961, S. 1- 33, hier S. 15.

13 E. Cassirer, Der Gegenstand, S. 23. 
„Tragödie der Kultur“ oder auch Hofmannsthal in seiner „Schrifttum“-Rede als Einheit oder als Bindung verstanden wissen wollten. Eine dauernde Sehnsucht nach solcher Bindung prägt dieses Konzept kultureller Semiose, dessen ,Soziales' sich erst einer veritablen Metaphysik der Kultur verdankt. Entkörperlichte Gesellschaft aber scheint dieser Bindung nicht fähig; Gesellschaft in diesem Sinne ist nicht Kultur, weil sie nicht Person ist. Kultur, das ist das der unruhigen Gesellschaft Entgegengesetzte, und, so schon Friedrich Nietzsche in „Menschliches. Allzumenschliches“ (1878), „nach dem Westen zu wird die moderne Bewegtheit immer größer. [...] Diese Bewegtheit wird so groß, daß die höhere Kultur ihre Früchte nicht mehr zeitigen kann. Es ist, als ob die Jahreszeiten zu rasch aufeinander folgten. Aus Mangel an Ruhe läuft unsere Zivilisation in eine neue Barbarei aus." $" 14$

Eine solche Vorstellung deklariert den sozialen Raum allerdings nicht per se, sondern nur in Zeiten (und als Ausdruck) gesellschaftlicher, Verkommenheit' zum Gegenteil der Kultur. Idealerweise geht es darum, diesen sozialen Raum als den Körper der einen Kultur wieder entstehen zu lassen. Man könnte auch sagen: Wo Gesellschaft ist, soll Kultur und das heißt: soll Gemeinschaft werden. Der kulturelle Raum ist nicht dispers, er ist der eine, vergrößerte Körper selbst.

Zurecht mag man bezweifeln, ob der Kulturbegriff heutiger Kulturwissenschaft(en) sich aus solchen Annahmen entwickelt hat. Ließe sich im Gegenteil nicht besser davon sprechen, dass dieser Kulturbegriff mit solcher Tradition brach und eine Art Import darstellt, der sowohl das, was im französischen Sprachraum als ,civilisation“ verstanden, als auch das, was im angelsächsischen Bereich als ,cultural studies' konzipiert wurde, ins Deutsche als ,Kultur' rückübersetzt? Bei dieser Rückübersetzung liegen die Voraussetzungen der ,cultural studies' dann in der europäischen Entwicklung einer vergleichenden Religionsgeschichte, die sich schon bald zur Ethnographie transformierte; sie liegen hingegen nicht so sehr in den zeitgleichen Varianten eines ,deutschen historischen Kulturbegriffs', wie er sich etwa in der Volkskunde und der ,Culturgeschichte' entfaltet hat - um dann aber doch auch mit diesen Konzepten eine eigentümliche Verbindung einzugehen.

V.

Bei der Entwicklung einer Ethnologie, die eng mit der Geschichte der Durkheim-Schule verbunden war, ging es sehr bald darum, Herders ,brutum' nun als ,Kultur', und zwar als kulturelle Variante oder als kulturellen Anfang, umzuinterpretieren. „Denn ein Barbar“, so später Claude Lévi-Strauss, ,ist ja vor allem derjenige, der an die Barbarei glaubt.“'15 Ganz unabhängig davon, ob man dieses ,brutum' als Spiegel früherer Zeit in die europäische Geschichte

14 Friedrich Nietzsche: „Menschliches. Allzumenschliches“ (1878), Nr. 285, in: Gesammelte Werke, Bd. 3, München o. J., S. 234.

15 Claude Lévi-Strauss: „Rasse und Geschichte“, in: Ralf Konersmann (Hg.), Kulturphilosophie, Leipzig 1996, S. 168-221, hier S. 177. 
rückzuholen bemüht war, als die Sichtbarmachung der Kindheit Europas selbst, ob man auf die Suche nach Typen menschheitsgeschichtlicher Entwicklung ging oder Kulturkreisthesen belegen wollte wie Aby Warburg ${ }^{16}$ die Praktiken indigener Gesellschaften galten als die Ausformung einer Sozialität, die als das Ergebnis von ,Kulturierung' verstanden wurde.

Marcel Mauss' berühmter „Essai sur le Don“, der Gabentausch, vollzieht dabei im Sinne der Durkheim-Schule eine folgenreiche Umpolung. Nicht nur stehen die kulturierenden Praktiken indigener Gesellschaften im Mittelpunkt, sondern deren Soziabilität wird auf Verfahren der verpflichtenden Wechselseitigkeit, des Reziproken, zurückgeführt. Diese Verfahren gelangen nie zur Ruhe, weil nicht so sehr die Materialität, sondern der ,Geist' der Gabe zurückzuerstatten ist, will man - in einem ganz wörtlichen Sinn - nicht als Schuldner nur ein Teil des Gebers sein. Damit wird das Reziproke zur Möglichkeit und Notwendigkeit, pneumatische und materielle Äquivalenz als entscheidende Bedingung des Sozialen zu denken. Nicht eine auf Dauer gestellte Asymmetrie wird zum Grundprinzip von Gesellschaft erklärt, sondern erst der Gabentausch als ,fait sociale totale', die Wechselseitigkeit der Gaben und die Vielschichtigkeit kultureller Praktiken ,ergeben' das Soziale, das ganz grundlegend mit der Vorstellung einer Umkehrung, dem Wechsel der Seiten, verbunden ist. Solche Reversibilität beruht auf einem kulturierenden Prozess und bringt ihn zugleich auch immer hervor.

Auf diese Weise - wie allmählich auch immer - wird eine Relativierung europäischer Gesellschaften als alleinigen Trägern der Kultur denk- und formulierbar. Dabei sieht man die Bewegung des ,von Innen nach Außen' nachhaltig verkehrt. Denn es geht nun weniger um den Akt geistigen Hervorbringens, an dessen Ende jene Kultur steht, die einem Nicht-Geistigen, Äußerlichen, ontologisch gegenüberliegt. Die Bewegung des ,von Innen nach Außen“ wird ersetzt durch eine Art ,Flächigkeit', ja ,Äußerlichkeit‘, die das Soziale und die Kultur im wesentlichen als eins denkt; sie untersucht die kulturierende Praxis unterschiedlicher Formationen in vergleichbarer Perspektive und führt auf diesem Wege zu einer Vervielfachung von Kultur zu den Kulturen.

Es wäre im einzelnen zu zeigen, wie sich mit einer Mauss'schen Ethnologie sowohl ein strukturalistisches Modell entwickeln als auch die historische Schule in bestimmten Punkten beerben lässt, dort nämlich, wo als methodologisches Prinzip der Blick auf das Konkrete gelenkt werden soll. Vergleich und Konkretes sollen eine Art menschheitsgeschichtliche Ausrichtung ergeben, die sich allerdings nicht mehr im Sinne des 18. Jahrhunderts versteht, sondern die Anerkenntnis von Verschiedenheit aus einer Analyse des Sozialen als einer Kultur der Wechselseitigkeit gewinnt. Dies etwa bei Claude Lévi-Strauss, der in seinem Vortrag „Rasse und Geschichte“ von 1952 eine Art Resümee zieht und Parameter für die Verschiedenheit von Kulturen aufstellt. Ohne seine Raum-, Geschichts- und Zeitkonzepte, seine stationären und kumulativen Phasen hier näher beleuchten zu können, ist doch der kategorische Imperativ des gesamten Theorieentwurfs von besonderer Bedeutung; er eröffnet sich am Ende als Credo:

16 Vgl. Aby Warburg: Schlangenritual. Ein Reisebericht (1923), Berlin 1988. 
„Das Faktum der Verschiedenheit [von Kulturen] ist zu erhalten, nicht der historische Inhalt, den jede Epoche ihm gegeben hat und den keine über sich verlängern kann. [...] man muß [...] bereit sein, ohne Überraschung, Abscheu und Empörung ins Auge zu fassen, was alle jene neuen sozialen Ausdrucksformen unweigerlich an Ungewohntem aufweisen werden. Toleranz ist keine kontemplative Einstellung, die dem, was war oder ist, mit Nachsicht begegnet. Es ist eine dynamische Haltung, die darin besteht, was sein will, vorauszusehen, zu verstehen und zu fördern. Die Verschiedenheit der menschlichen Kulturen ist hinter uns, um uns und vor uns. Die einzige Forderung, die wir in dieser Hinsicht erheben können (und die für jeden einzelnen entsprechende Pflichten schafft), ist, daß sie sich in Formen realisiere, von denen jede ein Beitrag zur größeren Generosität der anderen sei.“17

Solch’ 1952 eingeforderte Generosität aber beruht auf der Einsicht, dass es weder einen Nullpunkt der Geschichte noch die ,Reinheit‘ einer Kultur oder Rasse, sondern nur Mischverhältnisse gibt, in denen stets das vermeintliche Paradoxon zu bearbeiten versucht werde, Konvergenz und ein Optimum an Verschiedenheit zusammenzudenken. Oder, bei Lévi-Strauss etwas technischer formuliert: Es gebe immer nur die Gleichzeitigkeit zweier Bewegungen, die Annäherung des Verschiedenen und seine sofortige (Re-)Differenzierung.

Damit wird programmatisch bejaht, was etwa Hofmannsthal in seiner „Schrifttums“- Rede noch als den großen Sündenfall der Romantik dargestellt hatte: „das Musikmachen mit allem und aus allem.“18 ,Bindung' erscheint dem ethnologischen Blick nicht als metaphysisches Ziel oder Ausgangspunkt, sondern als nur temporär stabile Verknüpfung unterschiedlicher Elemente, als das Dritte, das den Prozess wechselseitigen Gebens und Nehmens zusammenschließt.

Denkt man das ,Soziale“ als kollektive Kulturierung im Prozess von Gabe und Gegengabe, so geht es stets um Interdependenz. Es ist daher kein Zufall, dass alle jene Theorien, die das Wort ,Zivilisation“ im Titel führen, etwa Norbert Elias” „Über den Prozeß der Zivilisation““19, an der Nachzeichnung solcher Interdependenzen interessiert sind und deren Zunahme zugleich als Verlauf moderner Gesellschaften zu bestimmen versuchen; dabei wird eine Bewegung beschrieben, die man bei Elias - im Unterschied zum Neuhumanismus - als ,von Außen nach Innen' bezeichnen könnte: die Umpolung von äußerer Gewalt in inneren Zwang korreliert für ihn mit der Ausweitung der Handlungsketten und der Veränderung von Lebensführung; die Affektkontrolle, die Internalisierung der Zwänge bildet zugleich komplexere gesellschaftliche Formen heraus usw. - alles dies Effekte wechselseitiger Abhängigkeiten, bei denen Verhalten sich durchmischt und eine Komplexierung der Spielarten ergibt. ,Umleitung' ist daher ein Stichwort, das schon in Elias' ZivilisationsBuch von 1936 eine zentrale Rolle spielt, ja eigentlich schon in Marcel

17 C. Lévi-Strauss: Rasse und Geschichte, S. 221.

18 Hugo von Hofmannsthal: „Das Schrifttum als geistiger Raum der Nation“, in: ders., Gesammelte Werke. Reden und Aufsätze III, Frankfurt/Main 1989, S. 2441, hier S. 36.

19 Norbert Elias: Über den Prozeß der Zivilisation, 2 Bde., Frankfurt/Main 1976. 
Mauss’ Essay von 1925 klar formuliert wird: „Es ist hier eine Art Hybride aufgeblüht.، “20

Von diesem Punkt aus führt auch eine Linie zu allen Theorien der Hybridität, die sowohl die ,race', ,class', ,gender'- Zentrierung vieler ,Cultural studies' grundiert als auch das, was - noch spezifischer - in Form der ,postcolonial studies' dann entwickelt wurde: Es gibt immer nur Mischungen, Hybridisierungen, den Prozess einer sehr komplexen, sich endlos verändernden kulturellen Semiose, es geht, wie der Medientheoretiker Marshall McLuhan dann in den 1950er Jahren formuliert, um „Energie aus Bastarden““21 - dies eine Konzeption, die bis in die Wissenschaftstheorie eines Bruno Latour zu verfolgen ist. $^{22}$

Solche ,Energie aus Bastarden', die durch die ,Innen-Außen'-Differenz nicht mehr beschreibbar ist, weil sie sich einer eindeutigen Zuordnung entzieht und stets etwas Drittes darstellt, operiert auch nicht mehr mit der Unterscheidung, die Kultur über die Differenz von Ware und Kunst bestimmt sah. ,Kultur' ist für einen Autor wie McLuhan eben beides, Ware und Kunst, und nicht nur eine sich dem Reich der Zwecke widersetzende, im Prozess des Herstellens authentische Arbeit, wie dies bei Adorno noch emphatisch gedacht wurde. Was als nicht mehr haltbar erscheint, ist das Konzept grundlegender Trennung selbst, mit dem Antagonisten benannt und in ihrem Widerspiel beobachtet werden können. Dies ist eine sehr einschneidende Veränderung, eben jene, die überhaupt erst die Beerbung des Kulturbegriffs durch ,cultural studies' (und vice versa) möglich gemacht hat. Denn diese ,cultural studies' sind im wesentlichen eine Verbindung der drei hier skizzierten Theoriestränge.

VI.

Nach der grundsätzlichen Unterscheidung zwischen ,Geist ${ }^{\star}$ und ,Kultur ' wurden mithin wichtige Differenzen dadurch eröffnet, dass die andere, meist verdunkelte Seite des Kulturbegriffs eine je unterschiedliche Benennung fand.

Der Text war von der Hypothese ausgegangen, dass es keine absoluten Begriffe, also keine Begriffe ohne Antonyme gibt. In diesem Sinne wurde erste Stufe - dem neuhumanistischen Kulturkonzept eine Vorstellung von ,Zivilisation' zugeordnet, die dem Versuch diente, ein inneres ,Aus-sich-heraussprechen' mit einem nur Äußerlichen zu konterkarieren, um das Verfahren dann in immer neuen Mischungen zu steigern. Dieses, Von-innen-heraus ${ }^{6}$ apostrophierte zugleich, setzte also ein zweites, drittes Innere voraus, das erwiderte, und in der (als Form mündlichen Austauschs gedachten) Geselligkeit

20 Marcel Mauss: „Die Gabe. Form und Funktion des Austauschs in archaischen Gesellschaften“, in: ders., Soziologie und Anthropologie, Bd. 2, hg. v. Wolf Lepenies/Henning Ritter, München, Wien 1975, S. 7-144, hier S. 131.

21 Vgl. Marshall McLuhan: „Energie aus Bastarden“, in: ders., Die Magischen Kanäle, Düsseldorf u. a. 1992, S. 65-73.

22 Bruno Latour: Wir sind nie modern gewesen. Versuch einer symmetrischen Anthropologie, Frankfurt/Main 1988. 
wahlverwandter Personen kam dann eine Sozialität zustande, die diejenigen, die ohne Eros waren, die, die nicht liebten, ausschloss.

,Um 1900` war - zweite Stufe - diese Differenz von Innen und, Äußerlichem' als ontologische Unterscheidung von ,innen und außen', Subjekt und Gesellschaft, Person und Natur sedimentiert. ,Kultur' erschien als der Sprachkörper einer Singularität, eines solipsistischen Subjekts, dessen ,symbolische Formen' mit ihm - wie eine Schrift - allein sind und das daher das Soziale als ,Bindung' immer nur in melancholischer Pose herbeisehnen konnte.

Mit dem Kulturvergleich, der in der frühen Ethnologie - dritte Stufe - einen ersten Höhepunkt erreicht, ziehen die ,Argonauten' dann aus, um sich selbst stets wiederzufinden, um in der Natur die Kultur zu entdecken und um die Kultur zu den Kulturen zu vervielfachen. Diesen Kulturen werden Prozesse der Äquivalenz, Reziprozität und Umkehrbarkeit unterstellt, und am Ende münden diese Annahmen in eine Theorie des Hybriden, der unablässigen Mischungen, die sich systematisch allen grundlegenden Trennungen entziehen. Mit der Idee totaler, unaussetzbarer Semiose verbunden, wird dann alles zur Kultur, weil ihr Anderes, ihr Gegenteil entweder fehlt oder aber in der Darstellung nicht mehr zur Erscheinung kommt.

In diesem Sinne formuliert Clifford Geertz:

„Ich [d. i. Clifford Geertz] meine mit Max Weber, daß der Mensch ein Wesen ist, das in selbstgesponnene Bedeutungsgewebe verstrickt ist, wobei ich Kultur als dieses Gewebe ansehe. Ihre Untersuchung ist daher keine experimentelle Wissenschaft, die nach Gesetzen [...], sondern eine interpretierende, die nach Bedeutungen sucht. Mir geht es um Erläuterungen, um das Deuten gesellschaftlicher Ausdrucksformen, die zunächst rätselhaft scheinen. ${ }^{\text {‘23 }}$

Bei deutlicher Fortwirkung nicht nur Max Webers, sondern auch Diltheys und Cassirers wird hier eine Formgebung gedacht, eine Textur, an der unendlich fortzuweben und die daher an keiner Stelle im Sinne einer Letztbegründung festzustellen ist.

Solche Form - nun nicht mehr allein das Privileg einer Auszeichnung als Kunst - lässt sich dann vielerorts sehen, und es entsteht eine unendliche Reihe von Untersuchungen, die mit der Semiose von Form und der Frage nach den Nachbarschaften einzelner Figurationen die Welt als Textur zu ihrem Gegenstand machen.

Dieser Entgrenzung liegt zum Grunde, dass solche Figurationen und die von ihnen hervorgebrachten Konzepte, um des Vergleichs und der elektrischen Erregung willen, auf keine Theorie der Gesellschaft rückbezogen werden, wie dies vorzugsweise in einer system- oder auch medientheoretischen Perspektive geschieht. Die Analysen der ,cultural studies' unterlaufen bewusst die als defizient erscheinenden Erklärungen kommunikationstheoretischer Gesellschaftsbeschreibungen, die im Rahmen bislang etablierter Disziplinen gegeben wurden und die im wesentlichen apersonale Prozesse in den Augenschein nehmen. ,Cultural studies' konterkarieren solche Ansätze mit einer

23 Clifford Geertz: Dichte Beschreibung. Beiträge zum Verstehen kultureller Systeme, Frankfurt/Main 1987, S. 9. 
Melange aus Ereignisgeschichte, Blicken auf Symbolaustausch, Habitus und Feldstrukturen, und was sie versprechen, ist eine , dichte Beschreibung'.

\section{VII.}

Damit ergibt sich in der Rede von Kultur, aber auch in der Rede von sozialen Räumen und kulturellen Praktiken eine eigenartige Gemengelage, die zunächst noch einmal in sieben Beobachtungen zusammengefasst werden soll:

Erstens ließ sich Kultur denken als das Ergebnis der Semiose einer europäischen, ja deutschen Person.

Zweitens erscheint das Soziale als das Ergebnis kollektiver Kulturierung, als Effekt von Gabe oder Recht, die auf der Möglichkeit des Reziproken, der Verkehrung der Seiten beruhen. Es geht um die Konstituierung kollektiver, ja totaler Körperschaften.

Die Analyse dieser Kulturierung gelangt damit drittens über die Singularitätsbehauptungen der irreduziblen Person hinaus - eigentlich aber nur, um sie auf der Ebene von Kollektiven zumindest teilweise wiedereinzuführen. Denn sie diskriminiert, um überhaupt etwas zu beobachten, und gewinnt so spezifische Einheiten. Und sie ermöglicht nach dieser Diskriminierung wieder eine Vergleichbarkeit zwischen unterschiedlichen Formen vollzogener Kulturierung.

Daher setzt viertens die Idee allgemeiner kultureller Semiosis, die sich von der Vorstellung einer Barbarei verabschiedet hat, zugleich die Pluralisierung von Kultur zu Kulturen durch.

Diese Kulturen stehen fünftens in Wechselwirkung und erzeugen auf diese Weise stets Vermischungen, Hybride. Das Hybride wird damit nicht zu einem Ergebnis, sondern ist immer schon der Ausgangspunkt der Analyse. Es lässt bewusst stabile Oppositionen hinter sich, und es ist nicht mehr an der Konstituierung oder Ausdrucksgebung von Person, sondern an der Analyse einer Formation orientiert.

Sechstens kann alles zum Gegenstand einer Formationsanalyse geraten, die den Anspruch erhebt, alles Soziale als Effekt von Formierung lesen und verstehen zu können. Kultur in diesem Sinne hätte dann keinen Gegenbegriff, kein Antonym, mehr.

Auch mit der Annahme von, Welt als Textur' differenziert aber siebtens jede konkrete Analyse wieder in eine spezifische Formation, die entweder differente Kollektivkörper untersucht oder aber Hybridformen auf unterschiedliche Parameter ,abtastet', eben jene, die als konstitutiv erachtet werden und die die Partizipation von Subjekten an der Formation zu steuern scheinen (etwa: race, class, gender).

Man könnte auch sagen, dass sich das daraus ableitbare Programm von ,cultural studies", so wie es etwa in der Birmingham School nach den Transformationen aus dem Marxismus und dann besonders in nordamerikanischen Versionen zur Erscheinung kam, als die Verbindung der Idee personaler Semiosis mit einem Konzept universell möglicher Partizipation verstehen lässt, 
als Versuch, die Generosität, die Lévi-Strauss gefordert hatte, auf alle Ethnien oder Gruppen auszudehnen. Spiegelbildlich verkehrt, geht es dabei zugleich um den Versuch, die Regularien von Partizipation und Ausschluss gerade im Blick auf ,kleine Sachen' oder in der von Bourdieu propagierten Analyse der Praktiken, des (figurativen) Gebrauchs herauszuarbeiten. ${ }^{24}$

Damit steht die Untersuchung jenes Konkreten im Mittelpunkt, die schon Marcel Mauss emphatisch gefordert hatte. Und nur so wird ja erklärlich, dass die historischen Wissenschaften sich auf die Erweiterung ihrer Struktur- oder Ereignisgeschichten durch eine kulturwissenschaftliche Perspektive eingelassen haben.

Will man die Implikationen dieses Ansatzes verstehen, so ist allerdings zu fragen, warum die Ethnologie von Marcel Mauss und anderen mit ihrer Analyse des Gabentauschs Praktiken beobachtet, die gerade personale Austauschverhältnisse ins Zentrum stellen. Führt der Gabentausch aus Gründen zu einer Vorstellung von ,Sozialität", die sich eher an der Gemeinschaft und nicht an dem, was die Soziologie dann als Gesellschaft herauszuarbeiten versucht hat, orientiert? ${ }^{25}$ Dieser Gemeinschaft wäre das Soziale dann wiederum vornehmlich die Ausdehnung des einen kollektiven Körpers, nicht aber der im wesentlichen anonyme Prozess enteigneter Kommunikation und sozialer Differenzierung. Diese Gemeinschaft ist mithin Dorf und nicht Stadt, Provinz und nicht Metropole. Dann aber ist apersonale ,Gesellschaft' das nicht explizierte Andere, das die Ethnologie des ,Gabentausch“ im Kern erst begründet.

Es ist durchaus zu fragen, ob die Analysen zu ,Kultur' im Rahmen von ,cultural studies' an dieser Personalitätsvorstellung auch immer teilhaben, ob also nicht der Aufweis von ,Form' oder ,Figuration' - gerade in der Erbschaft der Zeit ,um 1900` - in ,diskrete Einheiten' mündet, in eine ,Spezifikation', die die Form als Einheit vor dem Hintergrund (vor der Umwelt) eher entropisch erscheinender laufender Gesellschaftsprozesse konturiert. Ein Indiz dafür mag sein, dass funktionalistische Theoretiker der Gesellschaft, etwa Niklas Luhmann $^{26}$, programmatisch ohne einen begründenden Kulturbegriff auszukommen versuchen; sie ersetzen ihn durch ,Kommunikation“ und führen ,Kultur' dann wieder ein, um zu erklären, wie in einer dezidiert nicht personal geprägten Gesellschaft doch personale Zurechnung zustandekommt. ,Kultur ist hier eine Art Täuschung, eine Selbstzuschreibung von Subjekten oder Gruppen (auch noch der ,Nation'), um einen Abgrenzungsgewinn zu erzielen, der etwas Wiedererkennbares, der ,Individualität' sichert. Der Aufweis sol-

24 Vgl. Pierre Bourdieu: „Das kulturelle Unbewußte“, in: ders., Zur Soziologie der symbolischen Formen, Frankfurt/Main 1970, S. 117-124; Pierre Bourdieu: „Zur Genese der Begriffe Habitus und Feld“", in: ders., Der Tote packt den Lebenden (Schriften zur Politik und Kultur, 2), Hamburg 1997, S. 59-78.

25 Vgl. dazu den sehr guten Forschungsüberblick bei Beate Wagner-Hasel: Der Stoff der Gaben. Kultur und Politik des Schenkens und Tauschens im archaischen Griechenland, Frankfurt/Main, New York 2000, S. 27-76.

26 Vgl. Niklas Luhmann: „Kultur als historischer Begriff“, in: ders., Gesellschaftsstruktur und Semantik. Studien zur Wissenssoziologie der modernen Gesellschaft, Bd. 4, Frankfurt/Main 1995, S. 31-54. 
cher Orientierung an ,Personalität' erklärte auch, warum - nun andererseits so viele Anhänger der Kulturwissenschaften durchaus zurückhaltend gegen Gesellschaftstheorie sind, ja auch in der Geschichtswissenschaft die Analyse von Alltag oder ,Feldern “ programmatisch gegen Strukturgeschichte ins Argumentationsfeld geführt haben.

Damit ergibt sich eine eigenartige Ambivalenz, die sowohl die Programmatik als auch die wissenschaftliche Praxis von ,Kulturwissenschaft' prägt. Semiotisch gefasst - alles ist kulturelles Zeichen, es gibt kein Außerhalb wird einerseits in der Tat der Gegenstandsbereich von Kulturwissenschaft in jeder Analyse von Figuration erweitert, so dass am Ende nur noch ,die semiotische Welt' als Extensionsbereich übrig bleibt. Dies ist der grundsätzlichen Überlegung geschuldet, dass ,Kultur' Resultat unaussetzbarer semiotischer Prozesse ist, einer steten Fähigkeit zu ,Formgebung' sich verdankt, in der sich etwas ausspricht, dessen Spur man nachzugehen versuchen mag. Die ethnologische Arbeit, die die Differenzen zwischen Kultur und Natur in ihren Feldstudien irreversibel auflöst, weil sie jeden Nullpunkt verabschiedet und alles der Vergleichbarkeit ausgesetzt hat, unterstützt diese Überlegung gleichsam ,empirisch ${ }^{\text {- }}$ - es gibt in der menschlichen Geschichte (und man könnte auch sagen: in der menschlichen Wahrnehmung) kein ,jenseits von Kultur', sondern nur die stets neu gemischte Differenz einzelner kultureller Prozesse, die sich ständig selbst umschreiben.

Indem sie diese Unterstützung leistet, rekonstruiert aber diese ethnologische oder auch historische Analyse notwendigerweise Kulturen, also bei allen Mischungsverhältnissen diskriminierbare Einheiten und sehr spezifische Formen, die unterhalb dieses programmatischen Kulturaprioris wieder zu Trennungen führen, ja diese unweigerlich voraussetzen - und wenn sie auch keine ,Burgfreiheit' mehr reklamieren, so errichten sie doch wieder Burgen, indem sie Einheiten in Form kollektiver Körperschaften zusammenfassen.

Diesem Befund korreliert in der gegenwärtigen Diskussion eine gewisse Ratlosigkeit. Das Spannungsfeld von ,Kultur ‘ zu ,Kulturen“ überträgt sich nämlich sofort in den Unterschied zwischen der einen Kulturwissenschaft und den Kulturwissenschaften und dann in die Möglichkeit, die einzelnen Kulturwissenschaften selbst zu differenzieren. Wie steht die These von ,Kultur als Austausch von Zeichen' mithin zu den differenten Formen, als die ,Kultur' über Rekurrenzen analysiert wird und bei deren Untersuchung - trotz des Formaprioris - eine Reihe von Verfahren zur Anwendung kommen (müssen), die über einen figurativen Ansatz hinausgehen - diese Frage ist je und je zu stellen.

Im Umgang mit dieser Frage haben sich - im Rückbezug auf das System überkommener Disziplinen - zumindest drei unterschiedliche Konzepte von Kultur und Kulturwissenschaft herausgebildet.

Das erste Konzept ist orientiert an der Analyse von Figuration. Transdisziplinär angelegt werden Segmente der ,Welt als Textur ${ }^{6}$ behandelt, und in dieser Behandlung extendiert die kulturwissenschaftliche Arbeit. Dabei bemüht man die Archive sehr unterschiedlicher Institutionen oder wissenschaftlicher Disziplinen auf sehr unterschiedlichem Niveau, ohne sie aber noch aus- 
schließlich beliefern zu wollen. Der transdiziplinäre Ansatz tendiert dabei dazu, zunächst nicht mehr für ein überkommenes Fach zu schreiben, sondern bislang nicht gesehene Konstellationen und in ihnen die Rolle von Figuration zur Erscheinung kommen zu lassen und gerade aus diesem Bemühen selbst wieder einen Fachzusammenhang zu erzeugen. Systematisch ist hierbei das Problem zu lösen, für die sehr gedehnte, aufs Ganze gesehen zur Heterogenität neigende Extension wissenschaftliche Anschlusskommunikationen sicherzustellen, die den zunächst ingeniösen Einsatz in eine Kette mit nicht reißendem Faden überführt, also Referenz, Redundanz usw. erzeugt.

Das zweite Konzept operiert mit einer medialen Differenz, die als wichtigstes Unterscheidungskriterium genutzt wird, um den Extensionsbereich von ,Kultur' wiedereinzuschränken. Genau besehen handelt es sich um eine $\mathrm{Me}$ dium/Form-Differenz, mit deren Hilfe ein medial bestimmter Formbegriff (mit dann nachfolgenden Annahmen) ins Zentrum der Betrachtung gestellt wird. Dies kann zu den überkommenen Disziplinen zurückleiten, führt man doch dadurch - im Rückgriff auf eine mediale Hinsicht - nicht einfach nur die bekannten Aufmerksamkeiten auf Texte, Bilder usw. wieder ein und kommt dann doch wieder bei einer (erweiterten) Literatur-, Kunst-, Filmwissenschaft usw., aber nicht bei einer Kulturwissenschaft, auch nicht bei einer allgemeinen Medienwissenschaft an. Zwar genießt diese Option ohne Zweifel den Vorteil, überschaubarere Einheiten in disziplinärer, d.h. sowohl konzeptueller, sozialer, funktions- und leistungsbezogener Hinsicht anzubieten und damit die Reproduktion von Wissenschaften sowohl zu sichern als dann auch beschreiben zu können. Zugleich wird aber der Impetus kulturwissenschaftlicher Arbeit (oder auch ihre Fiktion), ein Feld transmedial und in allen seinen Ausformungen zum Gegenstand zu machen, d. h. Figuration umfassend in den Blick zu bekommen, schon vom Anspruch eher gedrosselt und damit zur Ausnahme.

Das dritte Konzept geht von Gesellschaft aus und untersucht als bestehend geltende soziale Gebilde auf den sie konstituierenden kulturellen Prozess, d. h. als umfassende, totale gesellschaftliche Tatsache. Es ist vornehmlich - sofern es nicht allgemeine Wissenschaftsforschung ist - an einem disziplinären $\mathrm{Zu}$ gewinn interessiert, d. h. es nutzt den programmatischen Blick auf Kulturierung, um sowohl die Extension des Gegenstandsbereichs als auch eine Analyse seiner Formierung vornehmen und damit über quantitative Verfahren hinausgehen zu können. Dies war etwa der Ausgangspunkt einer Geschichtswissenschaft, die auf die Untersuchung des Alltags setzte, oder einer Soziologie, die ein Feld sozialer Praxen als Kultur zu bestimmen versuchte (etwa in der Tradition Bourdieus). Forschungen dieser Art führen nicht zu neuen Disziplinen, sondern reichern entweder komplementär an oder sie versuchen, ein komplexeres Bedingungsgefüge für die Arbeit zugrundezulegen. Sie neigen deshalb zu Beobachtungen zweiter oder dritter Ordnung, und so sind sie dann elementar an Selbstbeobachtung interessiert. Unter diesen Prämissen kommt der Untersuchung von Wissenschaftskulturen systematisch Bedeutung zu.

Gesellschaftstheorie und kulturwissenschaftliche Orientierung liefen dies die hier vertretene These - seit dem Beginn des 20. Jahrhunderts parallel, ohne wirklich eine wechselseitig konstitutive Beziehung eingehen zu können 
(etwa bei Simmel). In der verstreuten, schwer zuordnenbaren Kommunikation der Gesellschaft übernähme die Rede von ,Kultur' und die Abgrenzung von ,Kulturen' dann die Funktion, wiedererkennbare Segmente auszugliedern, denen so etwas wie ,Stil' und ,Politik' zugeschrieben werden könnte - aber nur, um dann doch wieder im basso continuo des gesellschaftlichen Kommunikationsprozesses unterzugehen. Insofern ginge es um eine notwendige Komplementarität, bei der die unentwegte Bewegung gesellschaftlicher Kommunikation durch die Wiedererkennbarkeit von Form aufgehalten und für einen Moment als spezifisch und rekurrent zugleich beobachtet, eingefroren werden und so auch als politisches Gebilde überhaupt erst identifiziert werden kann.

Die Spezifizität einer Kultur im Ensemble differenter Kulturen ist daher die notwendige Fiktion, ohne die gesellschaftliche Kommunikation gar nicht zu beobachten wäre. Erst diese Fiktion macht die Annahme von Feldern möglich, an deren Erkenntnis wir unsere Beobachtung und unser Handeln ausrichten und die wir auf ihre (semiotischen) Praktiken, auf die Form ihrer Aussagen untersuchen können. Mag diese Fiktion auch prinzipiell unhaltbar sein, so ist sie dennoch für jedes Prozessieren von Aussagen notwendig, weil nur so neben der Differenz die Referenz als die zweite Seite der Kommunikation (als eben auch Zwei-Seiten-Form) zur Erscheinung gebracht werden kann. Erst beides bestimmt das ,Spiel' in der Wissenschaft vom Menschen, und hier liegt auch der Ausgangspunkt für eine ,Politik kultureller Praktiken', die die, wie auch immer durch Schnittmengen gebildeten, sozialen Räume fragmentiert, um daraus wieder einen Distinktionsgewinn und mit ihm Vorteilsnahmen abzuleiten.

\section{Literaturverzeichnis}

Bollenbeck, Georg: Bildung und Kultur. Glanz und Elend eines deutschen Deutungsmusters, Frankfurt/Main 1994.

Bourdieu, Pierre: „Das kulturelle Unbewußte“, in: ders., Zur Soziologie der symbolischen Formen, Frankfurt/Main 1970, S. 117-124.

Bourdieu, Pierre: „Zur Genese der Begriffe Habitus und Feld“, in: ders., Der Tote packt den Lebenden (Schriften zur Politik und Kultur, 2), Hamburg 1997, S. 59-78.

Cassirer, Ernst: „Der Gegenstand der Kulturwissenschaft“, in : ders., Zur Logik der Kulturwissenschaften, Darmstadt 1961, S. 1- 33.

Dilthey, Wilhelm: Einleitung in die Geisteswissenschaften. Versuch einer Grundlegung für das Studium der Gesellschaft und der Geschichte, Bd. 1, Leipzig, Berlin 1922.

Elias, Norbert: Über den Prozeß der Zivilisation, 2 Bde., Frankfurt/Main 1976.

Geertz, Clifford: Dichte Beschreibung. Beiträge zum Verstehen kultureller Systeme, Frankfurt/Main 1987.

Herder, Johann Gottfried: Sämtliche Werke, Bd. 22, hg. v. Bernhard Suphan, Nachdruck Hildesheim 1967. 
Heinz, Marion: „Kulturtheorien der Aufklärung: Herder und Kant““, in: Regine Otto (Hg.), Nationen und Kulturen. Zum 250. Geburtstag Johann Gottfried Herders, Würzburg 1996, S. 139-152.

Hofmannsthal, Hugo von: „Das Schrifttum als geistiger Raum der Nation“, in: ders., Gesammelte Werke. Reden und Aufsätze III, Frankfurt/Main 1989, S. 24-41.

Koselleck, Reinhart: Vergangene Zukunft. Zur Semantik geschichtlicher Zeiten, 4. Aufl., Frankfurt/Main 1985.

Latour, Bruno: Wir sind nie modern gewesen. Versuch einer symmetrischen Anthropologie, Frankfurt/Main 1988.

Lévi-Strauss, Claude: „Rasse und Geschichte“, in: Ralf Konersmann (Hg.), Kulturphilosophie, Leipzig 1996, S. 168-221.

Luhmann, Niklas: „Kultur als historischer Begriff“, in: ders., Gesellschaftsstruktur und Semantik. Studien zur Wissenssoziologie der modernen Gesellschaft, Bd. 4, Frankfurt/Main 1995, S. 31-54.

Mauss, Marcel: „Die Gabe. Form und Funktion des Austauschs in archaischen Gesellschaften“, in: ders., Soziologie und Anthropologie, Bd. 2, hg. v. Wolf Lepenies/ Henning Ritter, München, Wien 1975, S. 7-144.

McLuhan, Marshall: „Energie aus Bastarden“, in: ders., Die Magischen Kanäle, Düsseldorf u. a. 1992, S. 65-73.

Nietzsche, Friedrich: „Menschliches. Allzumenschliches“ (1878), Nr. 285, in: Gesammelte Werke, Bd. 3, München o. J.

Rickert, Heinrich: Kulturwissenschaften und Naturwissenschaften. Nach der 6. Aufl. von 1926, hg. von Friedrich Vollhardt, Stuttgart 1986.

Simmel, Georg: „Der Begriff und die Tragödie der Kultur“, in: Ralf Konersmann (Hg.), Kulturphilosophie, Leipzig 1996, S. 25-57.

Vico, Giambattisto: Die neue Wissenschaft über die gemeinschaftliche Natur der Völker. Nach der Ausgabe von 1744, übersetzt und eingeleitet von Erich Auerbach, Berlin 1965.

Wagner-Hasel, Beate: Der Stoff der Gaben. Kultur und Politik des Schenkens und Tauschens im archaischen Griechenland, Frankfurt/Main, New York 2000, S. 27-76.

Warburg, Aby: Schlangenritual. Ein Reisebericht, Berlin 1988. 